\title{
Caracterización de reacciones adversas a medicamentos en adultos mayores de 44 años en Bogotá, D.C., enero a diciembre, 2012
}

\author{
Marlén Chaves \\ Facultad de Medicina, Universidad Militar Nueva Granada; \\ Programa Distrital de Farmacovigilancia de Bogotá, Bogotá, D.C., Colombia
}

Introducción. El envejecimiento de la población en Colombia es una realidad. Los ancianos consumen más medicamentos que los jóvenes, por lo que están más expuestos a las reacciones adversas que estos puedan provocar.

Objetivo. Caracterizar las sospechas de reacciones adversas a medicamentos que se presentaron en los adultos mayores de 44 años en Bogotá, en el año 2012.

Materiales y métodos. Se llevó a cabo un estudio de farmacovigilancia que incluyó 470 reportes de reacciones adversas a medicamentos y problemas relacionados con su uso en adultos mayores de 44 años registrados en la base de datos del Programa Distrital de Farmacovigilancia de Bogotá.

Resultados. Se evaluaron 470 reportes de reacciones adversas y problemas relacionados con el uso de medicamentos en adultos mayores de 44 años. De estos, 432 reportes $(91,9 \%)$ correspondieron a la categoría de sospecha de reacción adversa a medicamentos y 28 (6\%), a la de problemas relacionados con su uso. La tasa de incidencia de eventos adversos notificados en Bogotá fue de 22,5 por 100.000 adultos mayores, la cual aumentó de forma directamente proporcional a la edad de los pacientes. El grupo farmacológico más reportado fue el de los antibacterianos, con 94 notificaciones. El sistema orgánico más comprometido fue la piel y sus anexos, con 21,2\% de los casos. En cuanto a la evaluación de la gravedad, el 69,5\% de las reacciones adversas fueron moderadas, y con relación a la causalidad, la mayoría, es decir, $45,8 \%$ de los reportes, se catalogaron como posibles.

Conclusiones. La caracterización de las reacciones adversas a medicamentos en adultos mayores de Bogotá es similar a la reportada en la literatura científica para este grupo de población.

Palabras clave: efectos colaterales y reacciones adversas relacionados con medicamentos, anciano; anciano de 80 o más años, antiinflamatorios no esteroideos, antibacterianos, farmacovigilancia.

doi: http://dx.doi.org/10.7705/biomedica.v35i1.2194

Characterization of adverse drug reactions in adults over $\mathbf{4 4}$ years of age in Bogota, JanuaryDecember, 2012

Introduction: In Colombia, aging of the population is a reality and elders consume more drugs than the young do. Consequently, they are more exposed to adverse drug reactions.

Objective: To characterize suspected adverse drug reactions in adults over 44 years of age in Bogota in 2012.

Materials and methods: We conducted a pharmacological surveillance study that included 470 reports of adverse drug events and associated problems with the use of drugs in adults over 44 years old included in the database of Bogotá's pharmacosurveillance program.

Results: We evaluated 470 reports of adverse drug events and associated problems with the use of drugs in adults over 44 years of age. From these, 432 reports (91.9\%) were classified as suspected adverse drug reactions and $28(6 \%)$, as associated problems with the use of drugs. The incidence rate for adverse drug events reported in Bogotá was 22.5 for every 100,000 elders, which increased in direct proportion to patients' age. The most frequently reported drug was antibacterials with 94 notifications $(20 \%)$. The organ system with the highest number of alterations was the skin and annexes with $21.2 \%$ of cases. Regarding severity assessment, $69.5 \%$ of adverse drug reactions were moderate, and as for causality, most adverse drug reactions were classified as possible, with $45.8 \%$ of the reports.

Conclusions: The characterization of adverse drug reactions in older adults in Bogotá is similar to that reported in the literature for this population age group.

Key words: Drug-related side effects and adverse reactions, aged; aged, 80 and over; anti-inflammatory agents, non-steroidal; anti-bacterial agents, pharmacovigilance.

doi: http://dx.doi.org/10.7705/biomedica.v35i1.2194 
El envejecimiento de la población mundial es un fenómeno demográfico debido al incremento en la esperanza de vida, lo que conlleva el aumento en el número de ancianos. Colombia no es ajena a este cambio en el perfil demográfico; se espera que para el 2050 el total de la población se acerque a los 72 millones, y que más del $20 \%$ esté por encima de los 60 años. El envejecimiento de la población no es homogéneo en todo el territorio nacional: el 63,12 \% de la población adulta mayor se concentra en Bogotá, Antioquia, Valle del Cauca, Cundinamarca, Santander, Atlántico, Bolívar y Tolima, siendo Bogotá una de las ciudades con mayor concentración de adultos mayores (1).

Este grupo de población en crecimiento usa un gran número de medicamentos en comparación con la población joven. Como lo señala la Organización Mundial de la Salud (OMS), los adultos mayores usan alrededor del doble de medicamentos que los adultos jóvenes, y no es raro que reciban simultáneamente cinco o seis prescripciones, en muchos casos de especialistas diferentes (2).

El uso de medicamentos tiene el riesgo inherente de los problemas relacionados con su utilización, definidos por el Tercer Consenso de Granada como "aquellas situaciones que en el proceso de uso de los medicamentos causan o pueden causar la aparición de un resultado negativo asociado a la medicación", así como los eventos adversos a medicamentos, entendidos como cualquier episodio médico desafortunado que pueda presentarse durante el tratamiento con un medicamento, pero que no tiene necesariamente una relación causal con ese tratamiento, y la reacción adversa a medicamentos, que la OMS ha definido como "una reacción nociva y no deseada que se presenta tras la administración de un fármaco, a dosis utilizadas habitualmente en la especie humana, para prevenir, diagnosticar o tratar una enfermedad, o para modificar cualquier función biológica". Nótese que esta definición implica una relación de causalidad entre la administración del medicamento y la aparición de la reacción. Este riesgo aumenta con la fragilidad del paciente y con la polifarmacoterapia (3). Las reacciones adversas

\footnotetext{
Correspondencia:

Marlén Chaves, Hospital Militar Central, Transversal $5 \mathrm{~N}^{\circ} 49$ 00, piso 4, edificio de la Facultad de Medicina, Bogotá, D.C. Colombia

Teléfono: 650 0000, extensión 2048

marlenchaveztdoc@yahoo.es

Recibido: 07/11/13; aceptado: 16/09/14
}

a medicamentos constituyen una causa importante de morbimortalidad de los pacientes, un reto para el profesional de la salud y un problema de salud pública para la ciudad y el país $(4,5)$.

Se ha encontrado que la hospitalización a causa de una reacción adversa a medicamentos es cuatro veces más frecuente en los ancianos que en los adultos jóvenes, lo que corrobora su condición de grupo vulnerable. Entre los factores que predisponen a estos eventos, se han descrito la disminución de la reserva funcional, los cambios en la farmacocinética y en la farmacodinamia asociados con el envejecimiento, y las enfermedades crónicas que requieren de múltiples tratamientos y esquemas farmacológicos $(6,7)$. La prevalencia de las reacciones adversas a los medicamentos en adultos mayores es alta a nivel mundial, pero en Colombia, y específicamente en Bogotá, aún no ha sido bien documentada.

El Programa Distrital de Farmacovigilancia de Bogotá, D.C., trabaja en pro del uso seguro de los medicamentos, recolectando las notificaciones de las reacciones adversas causadas por medicamentos y los problemas relacionados con estos, emitidas por las instituciones prestadoras de salud, los expendedores de medicamentos y la comunidad en general. Posteriormente, esta información se analiza y se envía de nuevo a las entidades que las han reportado; ya consolidada, se hace llegar al Invima (centro nacional de farmacovigilancia) que, a su vez, la transmite al Centro Colaborador de la OMS para la Farmacovigilancia Internacional, ubicado en Uppsala, Suecia. Este centro se identifica habitualmente como el Uppsala Monitoring Centre (UMC) y, desde 1968, es el encargado de gestionar una base de datos de ámbito mundial llamada VigiBase, la cual cuenta con las notificaciones de cinco millones de casos de sospechas de reacciones adversas a medicamentos provenientes de casi 100 países. La información contenida en VigiBase es compartida de forma gratuita con los países miembros y asociados del programa de la OMS (8). Colombia es miembro oficial de este programa desde el 2014 (9).

El objetivo de este trabajo fue caracterizar las sospechas de reacciones adversas a medicamentos que se presentaron en la población de adultos mayores de Bogotá, y que fueron reportadas al Programa Distrital de Farmacovigilancia, entre enero y diciembre de 2012. En dicha caracterización se identificaron los grupos farmacológicos y los fármacos que con mayor frecuencia estuvieron 
involucrados en dichos reportes, clasificando las reacciones adversas a medicamentos según su gravedad, mecanismo de producción, sistema de órganos afectados y grado de causalidad.

\section{Materiales y métodos}

Se hizo un estudio observacional, descriptivo, de corte transversal y farmacovigilancia pasiva, en el cual se utilizaron las notificaciones espontáneas de las reacciones adversas causadas por medicamentos y de los problemas relacionados con estos en pacientes mayores de 44 años, generadas por 31 instituciones prestadoras de servicios de salud (IPS) de Bogotá, no discriminadas por nivel de complejidad, y enviadas al Programa Distrital de Farmacovigilancia durante el periodo del estudio.

\section{Población y muestra}

Se estudió el total de notificaciones de reacciones adversas causadas por medicamentos y de problemas relacionados con estos reportadas en la base de datos del Programa Distrital de Farmacovigilancia de Bogotá, entre los meses de enero y diciembre de 2012: se incluyeron aquellas que involucraban a pacientes mayores de 44 años y se excluyeron los reportes pertenecientes a los menores de 45 años. En la mayoría de los estudios en adultos mayores, se incluyen solo a los mayores de 50 años por considerarlos como los de mayor riesgo de enfermar; sin embargo, en este estudio se tomó un grupo etario por debajo de los 50 años, teniendo en cuenta los datos del estudio de suficiencia del plan obligatorio de salud (POS) del 2010, el cual mostró que a partir de los 45 años la demanda de servicios de las personas en los servicios de salud se incrementa, lo que implica mayores costos para el sistema de seguridad social (10).

\section{Procesamiento de la información}

Las fuentes de información fueron los reportes de reacciones adversas causadas por medicamentos y de problemas relacionados con estos, enviados al Programa Distrital de Farmacovigilancia de Bogotá. La base de datos se digitó en el programa Excel $^{\circledR}$ y se depuró antes de ser analizada. Se analizó la frecuencia de cada variable discriminando por grupo etario y sexo; las tasas de incidencia se calcularon con base en la proyección de población del DANE para el año 2012 por grupo de edad.

Para la descripción de las reacciones adversas a medicamentos, se utilizó la terminología de la OMS (WHO - Adverse Reaction Terminology, ART), y los fármacos se describieron según la clasificación anatómica, terapéutica y química de los medicamentos de la misma organización (Anathomo-Therapeutic and Chemical Classification - ATC).

La sospecha de reacciones adversas se clasificó según el nivel de intensidad, así (11): leve, cuando no interfirieron con la actividad habitual del paciente, tuvieron corta duración, se resolvieron espontáneamente y no requirieron intervención de personal de salud ni prolongación del tiempo de hospitalización y, en general, no se requirió la suspensión del medicamento; moderada, cuando la reacción interfirió con la actividad habitual del paciente, requirió intervención de personal de salud para una mejor solución, aumentó el tiempo de estancia hospitalaria e implicó una modificación del tratamiento, aunque no necesariamente la suspensión del medicamento causante de la reacción; grave, cuando la reacción constituyó una amenaza para la vida del paciente, requirió hospitalización o su prolongación, la suspensión del medicamento causante de la reacción y la administración de un tratamiento específico para el manejo clínico de la reacción.

Según el mecanismo de producción, las reacciones adversas a medicamentos se clasificaron como: de tipo A, cuando representaban un cambio cuantitativo en la respuesta al medicamento (exageración del efecto farmacológico); de tipo B, cuando se relacionaban con un cambio cualitativo de la respuesta del paciente al medicamento, originando situaciones clínicas nuevas y diferentes a los efectos farmacológicos esperados; de tipo C, cuando su aparición se relacionaba con tratamientos farmacológicos prolongados; de tipo $D$, cuando estaban asociadas con mutagénesis o con anomalías del desarrollo embrionario o fetal (incluidas la dismorfogénesis o la teratogénesis); de tipo E, cuando aparecían como resultado de la suspensión súbita o repentina de la administración prolongada de un medicamento, y de tipo $F$, cuando se asociaban a la ausencia de respuesta clínica al medicamento o falla terapéutica (3).

La causalidad se evaluó utilizando el algoritmo de Naranjo y se categorizó así: probada (cierta o definitiva), cuando se presentaba una secuencia temporal razonable después de la administración del medicamento, había un patrón de respuesta conocido asociado con el medicamento sospechoso y se había confirmado una mejoría al suspender su administración, que reaparecía al administrarlo de nuevo sin que se pudiera explicar por otra 
causa; probable, cuando había una secuencia temporal razonable después de la administración del medicamento, así como un patrón de respuesta conocido asociado con este, una mejoría clínica razonable al suspender su administración, que no reaparecía al administrarlo de nuevo o ante una nueva exposición; posible, cuando había una relación temporal razonable después de la administración del medicamento, acompañada o no de un patrón de respuesta conocido asociado con su administración, la información relacionada con la suspensión del medicamento era incompleta o insuficientemente clara y la reacción podía explicarse por otra causa; improbable (dudosa o remota), cuando no había una secuencia temporal clara asociada con el medicamento sospechoso y la enfermedad de base, había otros medicamentos y químicos cuyo uso podía suministrar explicaciones razonables, por lo que la expresión clínica podría estar más relacionada con otros factores que con el medicamento presuntamente implicado, e inclasificable o no evaluable, cuando el reporte no podía evaluarse, pues la información disponible había sido insuficiente o contradictoria, y era imposible verificarla o complementarla con datos adicionales (12).

En cuanto a la falla terapéutica, esta se evaluó según el algoritmo del Centro de Información en Medicamentos de la Universidad Nacional de Colombia (CIMUN), el cual incluye las siguientes cinco categorías: 1) posiblemente asociado al uso inadecuado del medicamento por tratarse de medicamentos de farmacocinética compleja y estrecho margen terapéutico, que requieren manejo especial o cuyo uso inadecuado se podía documentar; 2) posiblemente en la efectividad de la intervención cuando se descartaron otras posibles causas que pudieran explicar la falla terapéutica y esta podía relacionarse con resistencia, tolerancia, resistencia o taquifilaxia; 3) notificación posiblemente inducida, cuando la notificación se refería explícita y exclusivamente a medicamentos genéricos; 4) posiblemente asociado a un problema 'biofarmacéutico' (calidad), cuando se habían descartado el mal uso del medicamento y las causas clínicas, y se encontraron inconformidades en el análisis de calidad, y 5) la categoría relativa a no tener información suficiente para el análisis (13).

\section{Consideraciones éticas}

El estudio se realizó teniendo en cuenta las recomendaciones internacionales y la normativa nacional vigente en materia ética (14-16). Las notificaciones de reacciones adversas causadas por medicamentos y de problemas relacionados con estos, no incluyen el nombre completo del paciente, solamente las iniciales, para mantener la confidencialidad de los datos; por ello, no se requirió consentimiento informado y el estudio no se sometió a consideración de un comité de ética por juzgarlo sin riesgo.

\section{Resultados}

Se evaluaron 470 reportes recibidos en el período de enero a diciembre de 2012 por sospecha de reacciones adversas a medicamentos y problemas relacionados con su uso en adultos mayores de 44 años, los cuales correspondieron al $24,6 \%$ del total de reportes recibidos en el Programa Distrital de Farmacovigilancia en ese año (1.907 reportes). De estos, 432 reportes $(91,9 \%)$ se catalogaron como sospecha de reacción adversa a medicamentos y, 28 (6\%), como problemas relacionados con el uso de medicamentos. La tendencia de la notificación varió a lo largo de los meses, con un ligero aumento en el número de reportes en marzo, julio y diciembre, sin que este fenómeno se pudiera atribuir a alguna circunstancia en particular (figura 1).

Al considerar la distribución por grupos de edad, se pudo observar que la mayor parte de los reportes correspondían a pacientes entre 50 y 64 años de edad (cuadro 1). La tasa de incidencia de las reacciones adversas causadas por medicamentos notificados en Bogotá fue de 22,5 por 100.000 adultos mayores y aumentó en forma directamente proporcional a la edad de los individuos (figura 2).

En la figura 3 se pueden apreciar los 10 grupos farmacológicos con mayor número de reportes

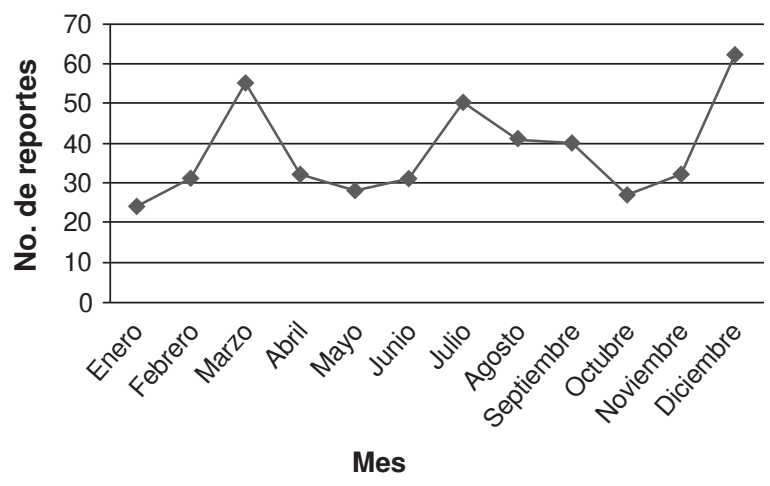

Figura 1. Tendencia del reporte de reacciones adversas causadas por medicamentos y problemas relacionados con estos en adultos mayores al Programa Distrital de Farmacovigilancia de Bogotá.

Fuente: base de datos del Programa Distrital de Farmacovigilancia de Bogotá, D.C. 
Cuadro 1. Frecuencia e incidencia de reacciones adversas causadas por medicamentos y problemas relacionados con estos según grupos de edad entre adultos mayores reportados al Programa Distrital de Farmacovigilancia de Bogotá, D.C., en 2012

\begin{tabular}{cccccccr}
\hline $\begin{array}{c}\text { Grupo de } \\
\text { edad (años) }\end{array}$ & $\begin{array}{c}\text { No. de } \\
\text { reportes }\end{array}$ & $\%$ & Hombres & $\%$ & Mujeres & $\%$ & $\begin{array}{c}\text { Tasa de incidencia de } \\
\text { RAM y PRM por 100.000 }\end{array}$ \\
\hline $45-49$ & 38 & 8,1 & 22 & 57,9 & 16 & 42,1 & 7,5 \\
$50-54$ & 75 & 16,0 & 24 & 32,0 & 48 & 64,0 & 16,7 \\
$55-59$ & 61 & 13,0 & 25 & 41,0 & 24 & 39,3 & 17,2 \\
$60-64$ & 76 & 16,2 & 33 & 43,4 & 43 & 56,6 & 28,3 \\
$65-69$ & 49 & 10,4 & 27 & 55,1 & 22 & 44,9 & 25,0 \\
$70-74$ & 58 & 12,3 & 30 & 51,7 & 26 & 44,8 & 43,2 \\
$75-79$ & 50 & 10,6 & 19 & 38,0 & 29 & 58,0 & 55,2 \\
80 o más & 63 & 13,4 & 21 & 33,3 & 39 & 61,9 & 70,2 \\
Total & 470 & 100,0 & $201^{*}$ & 42,8 & $247^{*}$ & 52,6 & 22,5 \\
\hline
\end{tabular}

RAM: reacciones adversas causadas por medicamentos; PRM: problemas relacionados con medicamentos

Fuente: base de datos del Programa Distrital de Farmacovigilancia de Bogotá, D.C.

*En 22 de los 470 reportes no se diligenció la variable sexo, por lo que no fue posible incluirlos en el grupo de hombres o de mujeres.

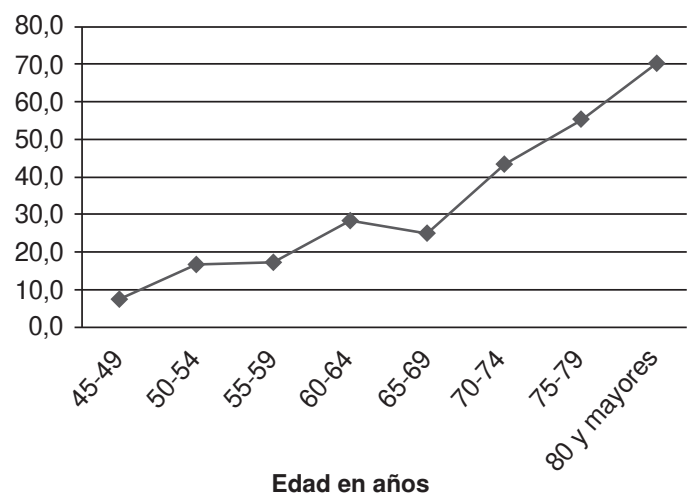

Figura 2. Incidencia de los reacciones adversas causadas por medicamentos y problemas relacionados con estos en adultos mayores reportados al Programa Distrital de Farmacovigilancia de Bogotá, D.C.

RAM: reacciones adversas causadas por medicamentos PRM: problemas relacionados con medicamentos

Fuente: base de datos del Programa Distrital de Farmacovigilancia de Bogotá, D.C.

de reacciones adversas y de problemas relacionados con su uso en adultos mayores. El grupo farmacológico más reportado fue el de los antibacterianos, con 94 notificaciones (20\%), seguido de los antiinflamatorios no esteroides, con 57 reportes $(12,1 \%)$. El primer lugar entre los medicamentos más reportados lo ocuparon la claritromicina y la warfarina, con 24 notificaciones $(5,1 \%)$ cada una, seguidas por la dipirona, con 19 reportes, es decir, el $4 \%$ del total de notificaciones.

En cuanto al comportamiento de las reacciones adversas a medicamentos, se notificaron 650 signos y síntomas en los diferentes sistemas orgánicos de los 432 adultos mayores afectados. En el cuadro 2 se presentan los 10 sistemas orgánicos involucrados con mayor frecuencia en

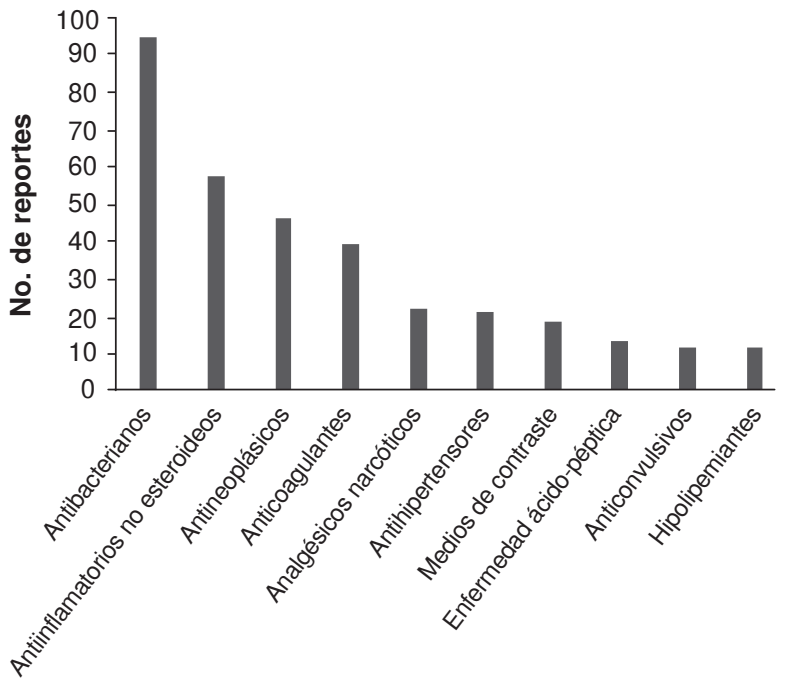

Grupo farmacológico

Figura 3. Los 10 grupos farmacológicos con mayor número de reportes de reacciones adversas causadas por medicamentos y problemas relacionados con estos en adultos mayores de Bogotá, D.C.

Fuente: base de datos del Programa Distrital de Farmacovigilancia de Bogotá D.C.

los reportes de sospecha de reacciones adversas a medicamentos; la piel y sus anexos fue el sistema más frecuentemente reportado, con 138 notificaciones (21,2\%). La flebitis, con 63 (14,7\%) notificaciones, fue la reacción adversa más reportada, seguida por la erupción cutánea y el exantema pruriginoso.

El comportamiento con respecto a la seriedad de las reacciones fue el siguiente: 292 reportes $(69,5 \%)$, es decir, la mayoría, correspondieron a reacciones moderadas; $76(18,1 \%)$ fueron graves y, $52(12,4 \%)$, leves. 
Cuadro 2. Los 10 sistemas orgánicos más comprometidos por reacciones adversas a medicamentos en adultos mayores de Bogotá, D.C.

\begin{tabular}{|c|c|c|}
\hline Sistema orgánico afectado & $\begin{array}{l}\text { Signos y síntomas } \\
\text { reportados }\end{array}$ & $\%$ \\
\hline Alteraciones de la piel y anexos & 138 & 21,2 \\
\hline Alteraciones generales & 113 & 17,4 \\
\hline $\begin{array}{l}\text { Alteraciones del sistema } \\
\text { gastrointestinal }\end{array}$ & 98 & 15,1 \\
\hline $\begin{array}{l}\text { Alteraciones vasculares (que no } \\
\text { son de origen cardiaco) }\end{array}$ & 72 & 11,1 \\
\hline $\begin{array}{l}\text { Alteraciones del sistema } \\
\text { nervioso central y periférico }\end{array}$ & 50 & 7,7 \\
\hline $\begin{array}{l}\text { Alteraciones del sistema } \\
\text { respiratorio }\end{array}$ & 42 & 6,5 \\
\hline Alteraciones psiquiátricas & 26 & 4,0 \\
\hline $\begin{array}{l}\text { Alteraciones de las plaquetas, } \\
\text { el sangrado y la coagulación }\end{array}$ & 21 & 3,2 \\
\hline $\begin{array}{l}\text { Alteraciones del ritmo y la } \\
\text { frecuencia cardiacas }\end{array}$ & 15 & 2,3 \\
\hline Alteraciones del sistema urinario & 13 & 2,0 \\
\hline Total & $588^{*}$ & 90,5 \\
\hline
\end{tabular}

Fuente: base de datos del Programa Distrital de Farmacovigilancia de Bogotá, D.C.

* Solo incluye los signos y síntomas que comprometieron los 10 sistemas orgánicos con mayor número de reportes

Con respecto al mecanismo de producción de la reacción adversa, las notificaciones correspondieron a los efectos de tipo A, B, C y F; no se notificaron reacciones adversas correspondientes a efectos de tipo $\mathrm{D}$ o $\mathrm{E}$, en tanto que los efectos de tipo $B$ fueron los más frecuentes, con 244 reportes $(56,5 \%)$, seguidos por los de tipo $A$, con 141 reportes (32,6\%), y hubo 25 reportes $(5,5 \%)$ de falla terapéutica $(F)$. Los medicamentos con mayor número de reportes de falla terapéutica fueron la bupivacaína, con cuatro, el propofol, con tres, y el ritonavir, la vancomicina y la warfarina con dos reportes cada uno. Los efectos de tipo $\mathrm{C}$ aparecieron en siete reportes $(1,65 \%)$ de reacciones adversas a medicamentos, en tanto que 15 de las 432 notificaciones de sospecha, no pudieron clasificarse según el mecanismo de producción.

Con relación a la causalidad (cuadro 3), la mayoría de las reacciones adversas a medicamentos, es decir, el $44,7 \%$ de los reportes, se catalogó como 'posible', mientras que para la falla terapéutica la categoría más reportada, con $40 \%$, fue la de 'posiblemente asociado al uso inadecuado del medicamento', representando el 2,3\% del total de las reacciones adversas, seguida por la de 'posiblemente en la efectividad de la intervención', con el $24 \%$ de los fallas terapéuticas.

\section{Discusión}

Teniendo en cuenta que los adultos mayores consumen más medicamentos que los adultos jóvenes y que la prevalencia de reacciones adversas aumenta en esta población, se podría esperar un mayor número de reportes $(17,18)$. Pero el método de notificación espontánea de reacciones adversas causadas por medicamentos tiene el problema del subregistro, ya que se estima que, comparado con la farmacovigilancia activa, con dicho método el personal de salud solo detecta entre el 5 y el $15 \%$ de los eventos (4). En Bogotá, los reportes de reacciones adversas causadas por medicamentos y de problemas relacionados con su uso en adultos mayores de 44 años representaron el $24,6 \%$ del total de reportes durante el 2012 , cifra que podría ser mayor considerando que el programa de farmacovigilancia del Distrito aún está en proceso de consolidación y no ha integrado al $100 \%$ de las instituciones prestadoras de servicios de salud (IPS) de la ciudad, y, además, el subregistro es alto y en la comunidad apenas comienza a desarrollarse la cultura del reporte de tales reacciones y problemas (19).

Según la revisión que se hizo, los trabajos de farmacovigilancia en ancianos están enfocados hacia adultos mayores de 50 y 60 años. En este estudio, no obstante, se tuvieron en cuenta a adultos mayores de 44 años de acuerdo con los grupos de edad considerados en el plan obligatorio de salud para la población colombiana. Se pudo

Cuadro 3. Distribución según categorías de causalidad de las reacciones adversas a medicamentos en adultos mayores en Bogotá, D.C.

\begin{tabular}{lrr}
\hline Categorías de causalidad de las RAM & $\begin{array}{c}\text { No. de } \\
\text { reportes }\end{array}$ & $\%$ \\
\hline Posible & 193 & 44,7 \\
Probable & 158 & 36,6 \\
Inclasificable & 45 & 10,4 \\
Definitiva & 9 & 2,1 \\
Improbable & 7 & 1,6 \\
Falla terapéutica & & \\
$\quad$ Posiblemente asociada al uso & 10 & 2,3 \\
$\quad$ inadecuado del medicamento & & \\
$\quad \begin{array}{l}\text { Posiblemente en la efectividad } \\
\text { de la intervención }\end{array}$ & 6 & 1,4 \\
$\begin{array}{l}\text { Posiblemente asociada a un } \\
\text { problema biofarmacéutico }\end{array}$ & 3 & 0,7 \\
$\quad$ Notificación posiblemente inducida & 1 & 0,2 \\
Total & 432 & 100,0 \\
\hline
\end{tabular}

RAM: reacciones adversas a medicamentos

Fuente: base de datos del Programa Distrital de Farmacovigilancia de Bogotá, D.C. 
observar que en el grupo etario comprendido entre los 45 y los 49 años, hubo un menor número de reportes, con el $8,1 \%$ del total de reportes en adultos mayores, siendo este comportamiento similar al de la población general, mientras que el grupo de 50 a 54 años duplicó el número de reportes, con el $16 \%$ del total (cuadro 1). Al calcular la tasa de incidencia de reacciones adversas causadas por medicamentos notificados en esta población, se apreció claramente que esta incidencia aumentaba con la edad (20).

Las enfermedades infecciosas ocurren a cualquier edad y es bien conocido que en los extremos de la vida los seres humanos son más vulnerables. Según su etiología, dichas enfermedades deben tratarse con antibacterianos, de ahí que estos medicamentos sean ampliamente utilizados y que, además de sus efectos terapéuticos, generen también reacciones adversas y problemas relacionados con su uso. Este comportamiento difiere de lo reportado en la literatura científica, en la que se documenta que los medicamentos cardiovasculares son los más frecuentemente comprometidos en los reportes de reacciones adversas a medicamentos para esta población específica $(21,22)$.

Los antinflamatorios no esteroideos son otro grupo farmacológico de uso frecuente en adultos mayores, con prescripción médica o sin ella, debido a la prevalencia de enfermedades osteomusculares como la artritis y la osteoartritis en esta población. Aunque estos fármacos son eficaces para controlar el dolor y la inflamación causados por dichas enfermedades, también generan reacciones adversas graves, entre ellas, la hemorragia digestiva, la cual encabeza la lista de causas de hospitalización por reacciones adversas a medicamentos en adultos mayores a nivel mundial. En este estudio, los antinflamatorios no esteroideos ocuparon el segundo lugar con el $12 \%$ de dichos reportes en Bogotá (23-25).

El sistema orgánico más afectado fue la piel, con $21,2 \%$ de las notificaciones, resultados que no difieren significativamente de lo reportado para la población general, en la cual las manifestaciones en la piel y sus anexos alcanzan el 25 a $30 \%$ de los casos. En cuanto a la gravedad, la mayoría de las reacciones adversas $(69,5 \%)$ se catalogó como moderada.En este punto es importante recordar que todas las notificaciones analizadas provenían de IPS de diferentes niveles de atención y no de la comunidad, por lo que existe la posibilidad de que las reacciones adversas leves, que corresponden a signos y síntomas que se resuelven espontáneamente, no lleguen a las IPS y, por lo tanto, no sean reportadas. Aun con esta salvedad, estos datos son similares a los reportados en otros estudios de farmacovigilancia, como el de Alves de Araujo en Brasil, entre otros (26-28).

En este estudio, la mayoría de las sospechas de reacción adversa se catalogó como posible según el algoritmo de causalidad de Naranjo, y representó el $45,8 \%$ de las notificaciones, porcentaje del cual solo el 2,1\% fue definitivo. Es importante anotar que la evaluación de la causalidad de las reacciones adversas a medicamentos depende en gran medida de la cantidad y la calidad de la información recopilada en las notificaciones espontáneas de reacciones adversas causadas por medicamentos y problemas relacionados con ellos, lo que permite imputarle la reacción adversa al medicamento sospechoso. Sin embargo, un número importante de los reportes analizados no estaban completamente diligenciados, lo cual dificultó la valoración de la causalidad.

Los hallazgos de esta investigación demuestran que la tasa de incidencia de reacciones adversas causadas por medicamentos y de problemas relacionados con estos en adultos mayores de 44 años notificados en Bogotá es de 22,5 por 100.000, cifra que debe tenerse en cuenta en los programas de salud pública dirigidos a esta población, ya que la mayoría de estos eventos son prevenibles. En general, el comportamiento de las reacciones adversas a medicamentos en cuanto a frecuencia, gravedad, tipo de reacción, causalidad y sistema orgánico afectado, es similar al reportado en la literatura científica para este grupo de población, siendo los antibacterianos y los antiinflamatorios no esteroideos los grupos farmacológicos con mayor número de reportes. El principal aporte de este trabajo ha sido dar a conocer la información local actualizada sobre reacciones adversas causadas por medicamentos y problemas relacionados con estos en una población específica de Bogotá, en este caso, adultos mayores, quienes se consideran vulnerables frente a este tipo de eventos. Con ello se busca incentivar el uso racional y seguro de los medicamentos en beneficio de la comunidad.

También, es de resaltar que, aunque la comunidad científica de esta ciudad ya está aceptando la cultura del reporte, hecho que se ve reflejado en las 1.907 notificaciones de reacciones adversas causadas por medicamentos y problemas relacionados con ellos recolectadas en el 2012 en 
el Programa Distrital de Farmacovigilancia, la notificación de las IPS aún es limitada y el número de reportes provenientes de la comunidad es bajo 0 inexistente.

Las limitaciones del estudio se derivaron de la falta de información en la mayoría de las notificaciones, lo que dificultó el análisis de los eventos y problemas causados por medicamentos y relacionados con su uso. Los reportes analizados en este trabajo provenían casi todos de IPS de segundo y tercer nivel de complejidad que cuentan con programas institucionales de farmacovigilancia organizados, pero la mayoría de las IPS de primer nivel de complejidad no cuentan con estos programas y no reportan este tipo de eventos al Programa Distrital de Farmacovigilancia, por lo cual quedaron por fuera del estudio, generando así un sesgo importante en la información.

Se recomienda fortalecer los programas institucionales de farmacovigilancia en las IPS, para mejorar la cantidad y la calidad de la información. También, es necesario llevar a cabo estudios sobre el uso de medicamentos en la población en general y en poblaciones específicas, como la de adultos mayores, que son asiduos consumidores de medicamentos.

\section{Agradecimientos}

Al Programa Distrital de Farmacovigilancia de Bogotá, D.C., por proporcionar los datos y los recursos necesarios para este trabajo.

\section{Conflicto de intereses}

La autora declara no tener conflicto de intereses.

\section{Financiación}

Este trabajo fue financiado por el programa Distrital de Farmacovigilancia de Bogotá, D.C.

\section{Referencias}

1. Fundación Saldarriaga Concha. Diagnóstico de los adultos mayores en Colombia, 2005. Fecha de consulta: 25 de junio de 2013. Disponible en: http://www.sdp.gov.co/portal/page/portal/ PortalSDP/SeguimientoPoliticas/Politicas\%20Poblacionales/ Envejecimiento\%20y\%20Vejez/Documentaci\%F2n/ A31ACF931BA329B4E040080A6C0A5D1C

2. Ruales M, Pelcastre B, Ángeles A. Medicamentos para adultos mayores: percepciones y adquisición en farmacias privadas de Morelos, México. Rev Chil Salud Pública. 2012;16:146-55. http://dx.doi.org/10.5354/0717-3652. 2012. 20299

3. Calderón-Ospina CA, Urbina-Bonilla A. La farmacovigilancia en los últimos 10 años: actualización de conceptos y clasificaciones. Logros y retos para el futuro en Colombia. Med UIS. 2011;24:47-63.
4. Ocampo J, Chacón J, Gómez J, Curcio C. Reacciones y eventos adversos por medicamentos en ancianos que consultan un servicio de urgencias. Colomb Med. 2008;39:135-46.

5. Passarelli MC, Jacob-Filho W, Figueras A. Adverse drug reactions in an elderly hospitalised population: Inappropriate prescription is a leading cause. Drugs Aging. 2005;22:767-77.

6. Petrovic M. Adverse drug reactions in older people. Drugs Aging. 2012;29:453-62.

7. Conforti A, Costantini D, Zanetti F, Moretti U, Grezzana M, Leone R. Adverse drug reactions in older patients: An Italian observational prospective hospital study. Drug Healthc Patient Saf. 2012;4:75-80. http://dx.doi.org/10.2147/DHPS. S29287

8. Organización Mundial de la Salud. Ser miembro del Programa Internacional de Farmacovigilancia de la OMS, 2010. Fecha de consulta: 4 junio de 2013. Disponible en: http://who-umc.org/graphics/24731.pdf.

9. Instituto Nacional de Vigilancia de Medicamentos y Alimentos. Notificación de reacciones adversas a medicamentos a Uppsala, 2012. Fecha de consulta: 4 junio de 2013. Disponible en: https://www.invima.gov.co/images/pdf/ farmacovigilancia_alertas/UPPSALA.pdf.

10. Ministerio de la Protección Social. Análisis del riesgo por concentración de población en grupos etarios mayores de 50 años en el Régimen Contributivo del Sistema General de Seguridad Social en Salud, 2011. Fecha de la consulta: 4 de junio de 2013. Disponible en: http://www.minsalud. gov.co/salud/POS/Documents/sitio/Analisis\%20riesgo\%20 concentraci\%C3\%B3n\%20poblaci\%C3\%B3n\%20 grupos $\% 20$ et $\%$ C3\%A1reos $\% 20$ mayores $\% 2050 \% 20$ a\%C3\%B1os\%20r\%C3\%A9gimen\%20contributivo.pdf

11. Valsecia M. Farmacovigilancia y mecanismos de reacciones adversas a medicamentos - 2000. Fecha de consulta: 4 de junio de 2013. Disponible en: http://med.unne.edu.ar/sitio/ multimedia/imagenes/ckfinder/files/files/13_farmacovigi.pdf.

12. Organización Mundial de la Salud. Comités de farmacoterapia. Guía práctica 2003. Fecha de consulta: 25 de junio de 2013. Disponible en: http://apps.who.int/medicinedocs/ pdf/s8121s/s8121s.pdf.

13. Vaca CP, De las Salas RP, López JJ, Sánchez R, Figueras A. Algorithm for the evaluation of therapeutic failure reports proposal and pilot analysis. Pharmacoepidemiol Drug Saf. 2013;22:199-206. http://dx.doi.org/10.1002/pds.3355

14. Asociación Médica Mundial. Declaración de Helsinki de la AMM - Principios éticos para las investigaciones médicas en seres humanos. Fecha de consulta: 26 de junio de 2013. Disponible en: http://www.unav.es/cdb/ammhelsinki2.html.

15. Ministerio de Salud. Resolución № 008430 de 1993. Fecha de consulta: junio 26 de 2013. Disponible en: http://www. unisabana.edu.co/fileadmin/Documentos/Investigacion/ comite_de_etica/Res_8430_1993_-_Salud.pdf.

16. Ministerio de la Protección Social. Decreto 2200 de 2005. Fecha de consulta: 26 junio de 2013. Disponible en: https:// www.invima.gov.co/images/pdf/medicamentos/decretos/ decreto_2200_2005.pdf.

17. Holguín E, Orozco J. Medicación potencialmente inapropiada en ancianos en un hospital de cuarto nivel en Bogotá. Universitas Médica. 2011;52:149-68. 
18. Cherubini A, Ruggiero C, Gasperini B, Aquila GD, Grazia M, Zampi E, et al. The prevention of adverse drug reactions in older subjects. Curr Drug Metab. 2011;12:652-7. http:// dx.doi.org/10.2174/138920011796504482

19. Hilmer SN, McLachlan AJ, Le DG. Clinical pharmacology in the geriatric patient. Fundam Clin Pharmacol. 2007;21:21730. http://dx.doi.org/10.1111/j.1472-8206.2007.00473.x

20. Comisión de Regulación en Salud. Acuerdo 030 de 2011. Fecha de consulta: 23 de mayo de 2013. Disponible en: http://www.alcaldiabogota.gov.co/sisjur/normas/Norma1. jsp? $\mathrm{i}=45258$.

21. D'Cruz S, Sachdev A, Tiwari P. Adverse drug reactions and their risk factors among Indian ambulatory elderly patients. Indian J Med Res. 2012;136:404-10.

22. Oscanoa TJ. Diagnosis of drug-related problems in elderly patients at the time of hospitalization. Rev Peru Med Exp Salud Pública. 2011;28:256-63. http://dx.doi.org/10.1590/ S1726-46342011000200012

23. O'Neil CK, Hanlon JT, Marcum ZA. Adverse effects of analgesics commonly used by older adults with osteoarthritis: Focus on non-opioid and opioid analgesics. Am J Geriatr Pharmacother. 2012;10:331-42. http://dx.doi.org/10.1016/j. amjopharm.2012.09.004
24. Roth SH, Fuller P. Pooled safety analysis of diclofenac sodium topical solution $1.5 \%(\mathrm{w} / \mathrm{w})$ in the treatment of osteoarthritis in patients aged 75 years or older. Clin Interv Aging. 2012;7:127-37. http://dx.doi.org/10.2147/CIA. S30884

25. Doña I, López N, Torres MJ, García J, García-Núñez I, Gómez F, et al. Drug hypersensitivity reactions: Response patterns, drug involved, and temporal variations in a large series of patients. J Investig Allergol Clin Immunol. 2012;22:363-71.

26. Lobo MG, Pinheiro SMB, Castro JG, Momenté VG, Pranchevicius CS. Adverse drug reaction monitoring: Support for pharmacovigilance at a tertiary care hospital in Northern Brazil. BMC Pharmacol Toxicol. 2013;14: 2-7. http://dx.doi.org/10.1186/2050-6511-14-5.

27. Cuéllar IC, Rodríguez EC. Reacciones adversas a medicamentos. Fecha de consulta: 23 de abril de 2013. Disponible en: http://med.javeriana.edu.co/publi/vniversitas/ serial/v44n4/0040\%20reacciones.pdf.

28. Dang A, Bhandare P. The profile of voluntary reported adverse drug reactions at a tertiary care hospital: A fifteen month prospective study. J Clin Diagn Res. 2012;6:1504-9. http://dx.doi.org/10.7860/JCDR/2012/4340.2544 